\title{
Propuesta de una interfaz de comunicación para la atención a las personas con discapacidad
}

\section{Proposal of a communicative interface for the attention of people with disabilities}

\author{
Sylvana Karla da Silva de Lemos Santos ${ }^{1}$, Joaquim Alves Costa Júnior ${ }^{1}$, Jaspion Leone Rocha ${ }^{1}$, \\ Danilo Freire Holanda de Paiva ${ }^{1}$ \\ 'Instituto Federal de Brasília, Brasil \\ sylkarla@gmail.com,joaquimjunior13@gmail.com,jaspion.rocha@ifb.edu.br,danilo.f.h.paiva@gmail.com
}

(Recibido: 26 de mayo de 2018; aceptado: 15 de junio de 2018)

\begin{abstract}
Resumen: Este trabajo discurre sobre una experiencia en el desarrollo de un prototipo de interfaz computacional para el suministro de información al usuario, incluida la persona con discapacidad. Se describen las versiones del prototipo, que se encuentra en marcha y posibilitó conocer mejor las necesidades de información de los usuarios por medio de pruebas. Para el diseño de una interfaz accesible, se deben comprender las formas de interacción de los diferentes usuarios, sean visuales, auditivas o táctiles. La propuesta fue financiada a través de un proyecto de innovación y contó con la participación de dos estudiantes de graduación en Ciencia de la computación del Instituto Federal de Brasilia (IFB). Los resultados muestran que las diferentes opciones de interacción posibilitan la autonomía del usuario y la accesibilidad.
\end{abstract}

Palabras clave: prototipo; persona con discapacidad; accesibilidad.

\begin{abstract}
This work discusses an experience with the development of a computer interface prototype for the provision of information to the user, including the person with disabilities. The versions of the prototype (which is underway) are described. Through tests, it widened the understanding of users' needs of information. For the design of an accessible interface one must understand the forms of interaction of the different users, be it visual, auditory or tactile. The proposal was funded by the innovation project and participated two undergraduate students in computer science from the Federal Institute of Brasilia (IFB). The results show that different interaction options allow for user autonomy and accessibility.
\end{abstract}

Keywords: Prototype; person with disability; accessibility.

\section{Introducción}

La divulgación de información en formato impreso ha sido sustituida, cada vez más, por el medio digital. Los canales de comunicación virtual, como Facebook y los sitios institucionales, vienen siendo utilizados por instituciones públicas (Instituto Federal de Brasília, 2017b), como el Instituto Federal de Brasilia (IFB), que utiliza estos canales para divulgar informaciones sobre los procesos de selección, los cursos ofrecidos, eventos, entre otros. Además de estos, también es común que las personas busquen información presencialmente, en los campi del IFB. Estas personas son de diferentes perfiles sociales y niveles de escolaridad, incluidas personas con 
discapacidad. Es común que la recepcionista del campus dependa de la primera persona con quien se busca un contacto presencial y, aunque exista esa gama de canales y formas de comunicación, muchas veces el usuario de la información no tiene éxito y, consecuentemente, su necesidad de información no es satisfecha.

Para viabilizar el acceso a la información divulgada por el IFB, se ha concebido la idea de construir una interfaz de comunicación con el usuario del IFB como una forma de proporcionar respuestas a las preguntas a menudo buscadas y, así, atender de forma amplia las necesidades de información de las personas. La propuesta está justificada por la creciente demanda de la población del Distrito Federal, en Brasil, que busca formación técnica y tecnológica profesional en el IFB (Instituto Federal de Brasília, 2017a), como muestra la Figura 1, así como por informaciones más uniformes y accesibles para atender a las personas con necesidades específicas y ayudar en el uso de las tecnologías de la información y de la comunicación (TIC).

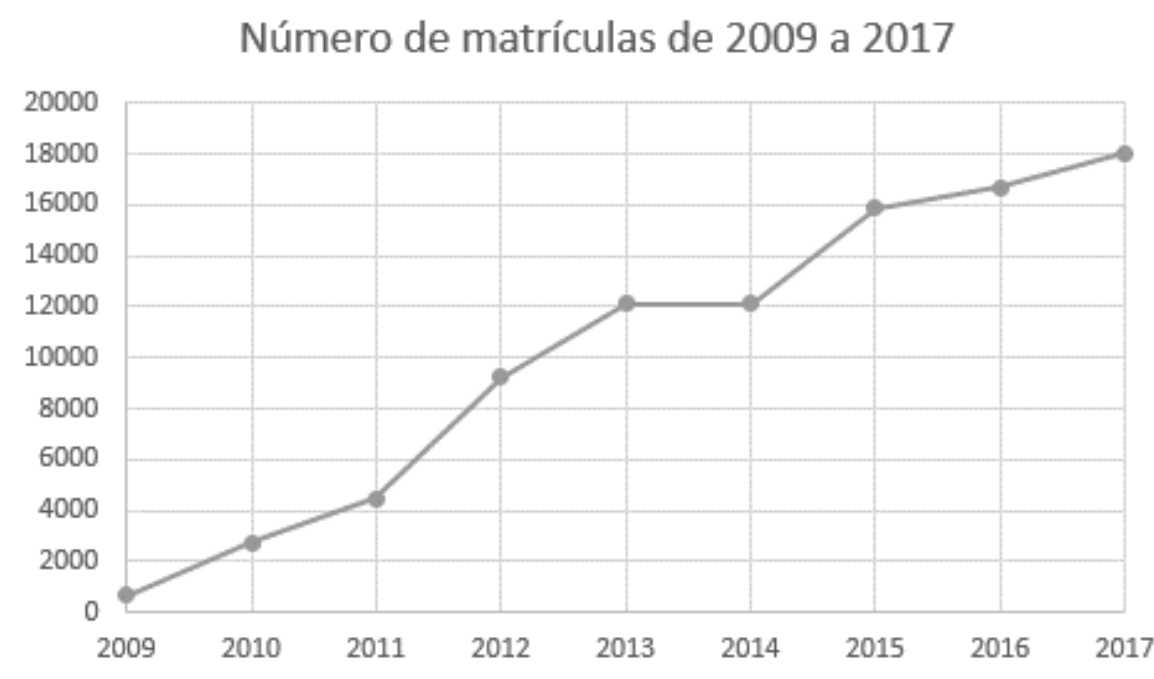

Figura 1. Cantidad de matrículas en el IFB de 2009 a 2017

Fuente: IFB en números. Acceso: 28 de noviembre de 2017

El objetivo de esta propuesta es, por lo tanto, desarrollar una interfaz de comunicación con el usuario que busca informaciones sobre el IFB, para atenderlo de forma actualizada, con accesibilidad y autonomía. Para ello, los objetivos específicos son: caracterizar las necesidades de información de los potenciales usuarios de la interfaz; identificar un formato de interfaz accesible para atender la satisfacción de los usuarios que buscan la información; realizar pruebas con los usuarios en la interfaz propuesta y, finalmente, poner a disposición la información de acuerdo con las necesidades de los usuarios.

Según la Ley 13.146/2015 (Presidência da República, 2015), la accesibilidad se caracteriza por la

posibilidad y condición de alcance para uso, con seguridad y autonomía, de espacios, mobiliario [...] información y comunicación, incluidos sus sistemas y tecnologías, así como de otros servicios e instalaciones abiertos al público, de uso público o privado de uso colectivo [...] por persona con discapacidad o con movilidad reducida.

En este sentido, de acuerdo con la referida ley, la presente propuesta se enmarca también como una tecnología asistiva, en la medida en que sirve como una estrategia de promoción de la funcionalidad, relacionada con la actividad y la participación de las personas con discapacidad o movilidad reducida, con vistas a su autonomía, independencia, calidad de vida e inclusión social.

Los bancos utilizan una interfaz digital que busca utilizar un lenguaje accesible, como forma de programación de servicios, algunas veces con la identificación del cliente a través de la agencia y de la cuenta para dirigir a una cola específica, ordenándolo por un número secuencial, preferencial o normal. De forma similar, los laboratorios de análisis clínicos hacen uso de una interfaz que dirige a sus clientes para la atención requerida. 
En bibliotecas, como la Biblioteca Central de la Universidad de Brasilia (UnB), la interfaz está orientada a la prestación de servicios sobre el funcionamiento de la biblioteca, consulta de acervo, entre otros servicios.

Como se ve, las interfaces se consideran canales alternativos de comunicación entre el usuario y el establecimiento, pues hay que considerar aquellos usuarios acostumbrados al contacto presencial y que prefieren buscar la información directamente con una persona.

La versión inicial del prototipo de la interfaz fue concebida a principios de 2016, a partir de la observación de los visitantes del Câmpus Gama y del tipo de información que buscaban, lo que se denomina comportamiento informacional (Ramalho, Hamad y Guimarães, 2016). Esta versión predijo, como requisito mínimo, la promoción de la comunicación prevista principalmente a través de texto escrito en portugués, audio y libras. La opción de audio pretende atender a las personas con discapacidad visual o ciegas y la ventana en libras - lengua brasileña de señales-, posibilitando que la información esté disponible en la lengua de signos. El texto de la escritura tiene como objetivo atender a aquellos que prefieren leer portugués en la navegabilidad, guiados por hipervínculos. Para la codificación se debe utilizar el lenguaje de programación Java. Su instalación física prevé el uso de una tableta apoyada sobre un tótem de 1 metro de altura con una inclinación similar a la presentada en la Figura 2, para facilitar la visualización de la información. El usuario tendrá un auricular disponible para ser utilizado, si desea hacer uso de la opción audio.
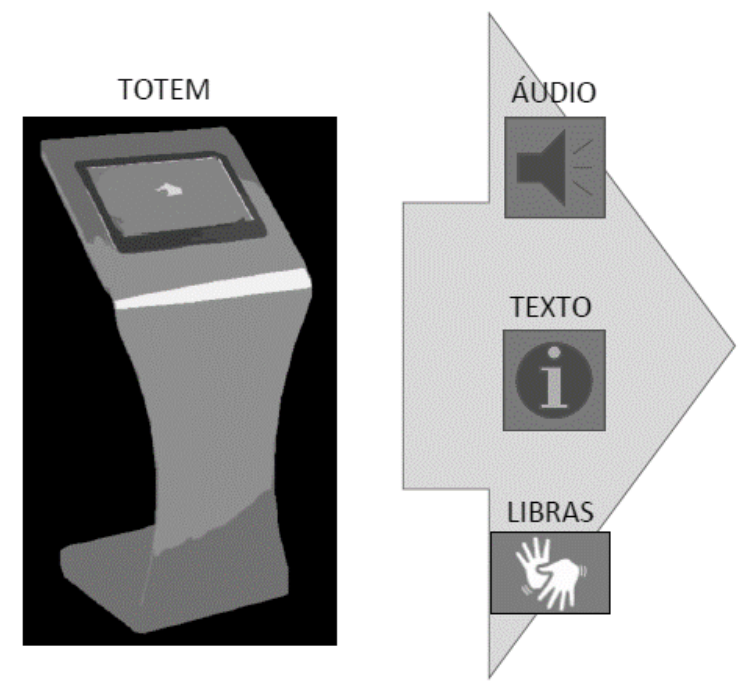

\section{USUÁRIO}

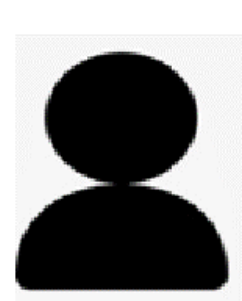

Figura 2. Esquema de funcionamiento de la interfaz con el usuario

\section{Metodología}

A continuación, se describirá el camino recorrido desde la primera versión hasta el actual prototipo de la interfaz de comunicación.

\subsection{Primera versión del prototipo}

La idea inicial del prototipo de interfaz fue desarrollado con la ayuda de un programa comercial para generar la presentación y se dio a conocer en septiembre del año 2016 durante el Foro Bilingüe Libras-Portuguesa (Instituto Nacional de Educação de Surdos, 2017), patrocinado por el Instituto Nacional de Educación para Sordos (INES), ubicado en Río de Janeiro, Brasil. La Figura 3 ilustra, de forma bastante simplificada, la idea desarrollada con la navegabilidad por hipervínculos. La pantalla se divide por la mitad: el lado izquierdo es el contenido escrito en portugués y, en el lado derecho de la pantalla, se encuentra la ventana libras, donde se encuentra el intérprete de 
libras para hacer el signo de los contenidos.

\title{
Instituto Federal de Brasília
}

\author{
Bem vindo(a) ao IFB! \\ Você está no campus \\ Gama. \\ Escolha uma das opçőes a seguir para \\ saber mais: \\ - $\underline{\text { Cursos }}$ \\ - Biblioteca \\ - Assistência ao Estudante \\ - Outros campi
}

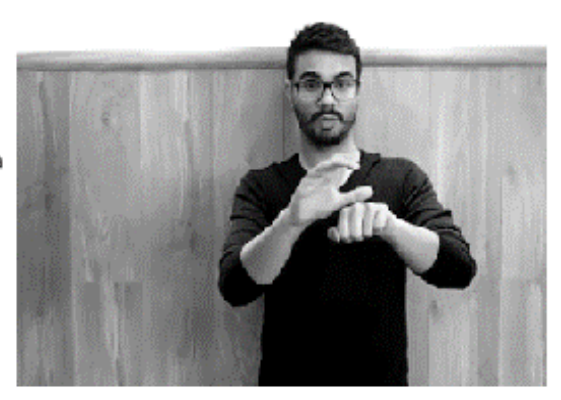

Figura 3. Pantalla principal de la primera propuesta de interfaz

Esta versión fue desarrollada con el propósito de proporcionar información diversa para aquellos que buscaban el Campus Gama, uno de los 10 campi actualmente en actividad del IFB.

La Figura 4 indica el diagrama de navegación en la aplicación. El ejemplo muestra el formato de la información en libras, seguido por la elección por los cursos disponibles. Al elegir la modalidad "técnico" se muestra al usuario los tipos de cursos ofrecidos por aquel campus.

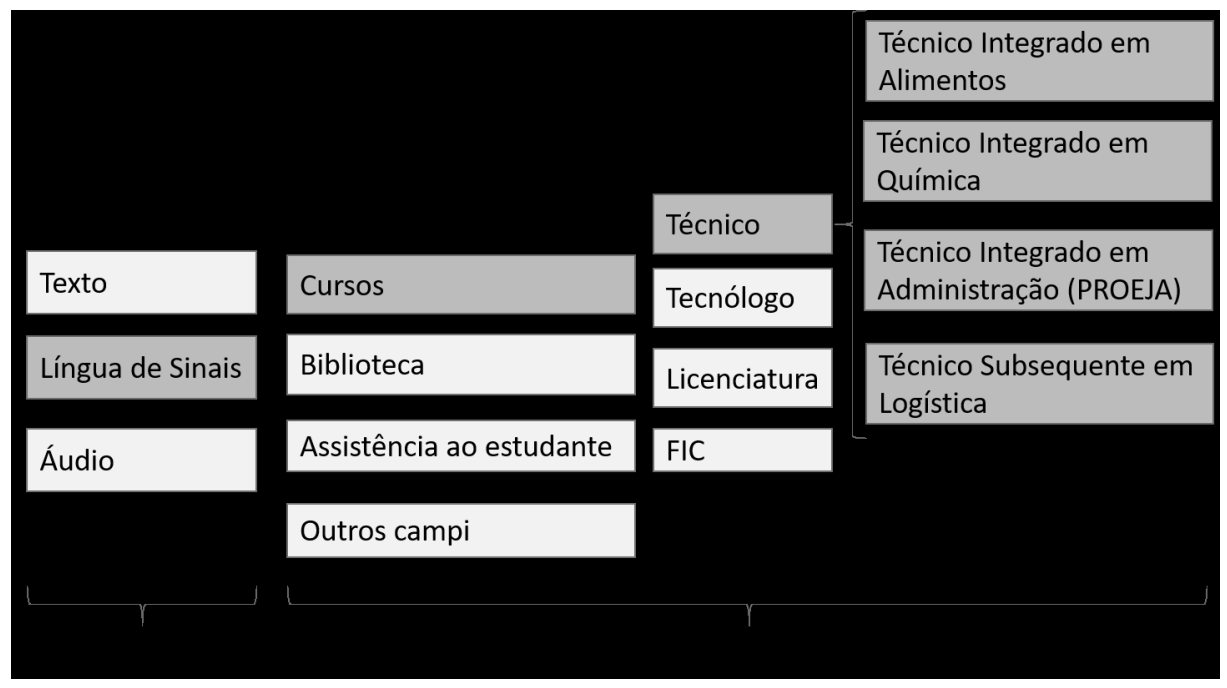

Figura 4. Diagrama de navegación en la primera propuesta de interfaz 
Para iniciar la interacción, la interfaz da la bienvenida al usuario y solicita que se escoja una de las opciones disponibles. Como ejemplo, el hipervínculo "cursos" fue explorado y, al ser pulsado, deberá exhibir, en la siguiente pantalla (Figura 5), las categorías de los cursos que son ofrecidos por dicho campus. A medida que se hace clic en cada categoría, la pantalla se cambia al contenido correspondiente, como el nombre del curso, la duración en semestres, la cantidad de vacantes y el inicio de las clases.

\subsection{Segunda versión del prototipo}

En el caso de las personas con baja visión, la segunda versión de la interfaz, mostrada en la Figura 6, incluye la opción de contraste de pantalla (fondo negro y fuente blanca) y la navegación por íconos, como avanzar y retroceder. Estos se acogen al modelo de accesibilidad del Gobierno electrónico (Ministério do Planejamento Orçamento, Secretaria de Logística, \& Tecnologia da Informação, 2014) y son más intuitivos para la mayoría de los usuarios, ya que se utilizan con frecuencia en otras interfaces digitales, como en aplicaciones móviles.

\section{Instituto Federal de Brasília}

\section{CURSOS}

O campus Gama tem cursos nos níveis:

- Técnico

- Tecnológico

- Licenciatura

(escolha uma das opções

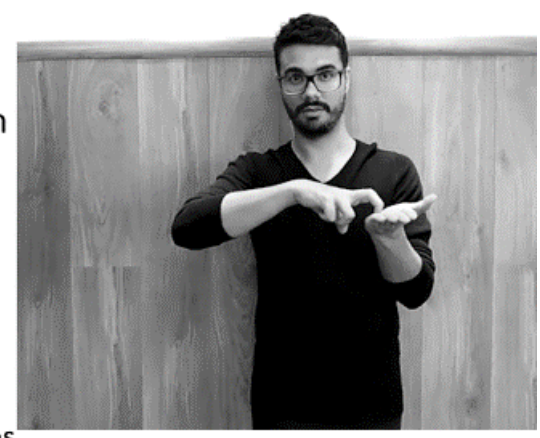
para prosseguir)

Figura 5. Opciones de cursos de la primera propuesta de interfaz

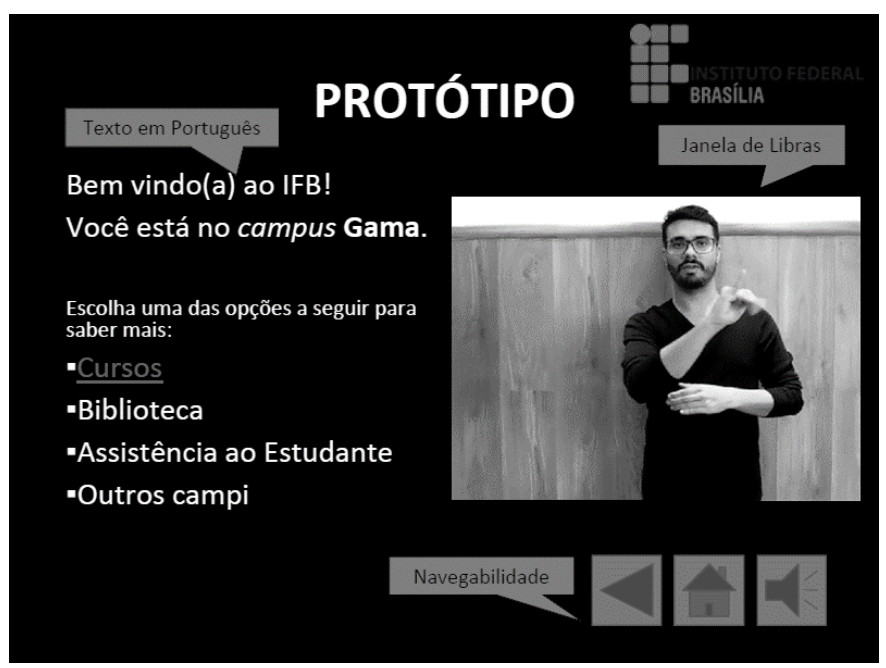

Figura 6. Pantalla principal de la segunda propuesta de interfaz 
Como principales características de esta versión se tienen: lenguaje claro y objetivo, con textos cortos; opciones de formatos: visual (texto e imagen), sonoro (audio) y lengua de señales (libras); posibilidad de contraste de pantalla y fuente ampliada para auxiliar a las personas con baja visión; navegabilidad simplificada, con el uso de iconos universales; e información personalizada para cada una de las 10 cámaras.

\subsection{Tercera versión del prototipo}

La evolución de la interfaz demuestra la necesidad de adaptación a las características que los usuarios suelen demandar. La movilidad que los dispositivos ofrecen, sean tablets o smartphones, se convierte en un requisito obligatorio en el desarrollo de la propuesta. En este sentido, una propuesta de innovación fue sometida al edicto de la Fábrica de Ideas Innovadoras (FABIN), a partir de la cual sería posible el financiamiento para la promoción del desarrollo del prototipo, denominado "Accesibilidad: información para TODOS".

La propuesta fue aceptada y, a partir de agosto de 2017, con la participación de dos becarios de graduación del curso Bachillerato en Ciencia de la computación del IFB, fue posible dar marcha a la codificación de la interfaz. Este aumento en el equipo posibilitó el desarrollo de la tercera versión del prototipo, utilizando el lenguaje Java orientado a la plataforma Android.

La idea es proporcionar un servicio de información que pueda incluir otras modalidades de interacción con el usuario, como audio y escritura, dándole más autonomía y satisfacción. Al atender a las personas con discapacidad a través de diversas opciones de formato, también se buscó atender las necesidades de las personas con dislexia o aquellas con dificultades de lectura textual, al hacer disponible información en audio, por ejemplo. Así, el título del proyecto, que incluye la palabra TODOS (en letras mayúsculas), viene a enfatizar la propuesta de una interfaz que posibilite el acceso a todos los individuos, o la mayoría de éstos.

Además de las cuestiones ya descritas en cuanto a la interfaz digital, también se propuso la adhesión de algunos mecanismos accesorios para auxiliar a las personas en general. Estos mecanismos son: piso táctil hasta el equipo, para posibilitar que personas con baja visión o ciegas puedan localizarse para encontrar el equipo; altura adecuada del tótem o aun sillones, para que las personas con estatura más baja, puedan tener acceso a la pantalla del equipo; sistema de sensores para identificar cuando el usuario se posiciona delante del equipo; control de la interfaz, tanto por voz como por botones; botones con escritura en Braille para lectura táctil de las funciones de navegación; auriculares y caja de sonido para que el usuario escuche la información; micrófono para controlar la interfaz de voz.

La Figura 7 muestra la versión actual del prototipo con la presentación de las opciones de información en texto, libras y audio.

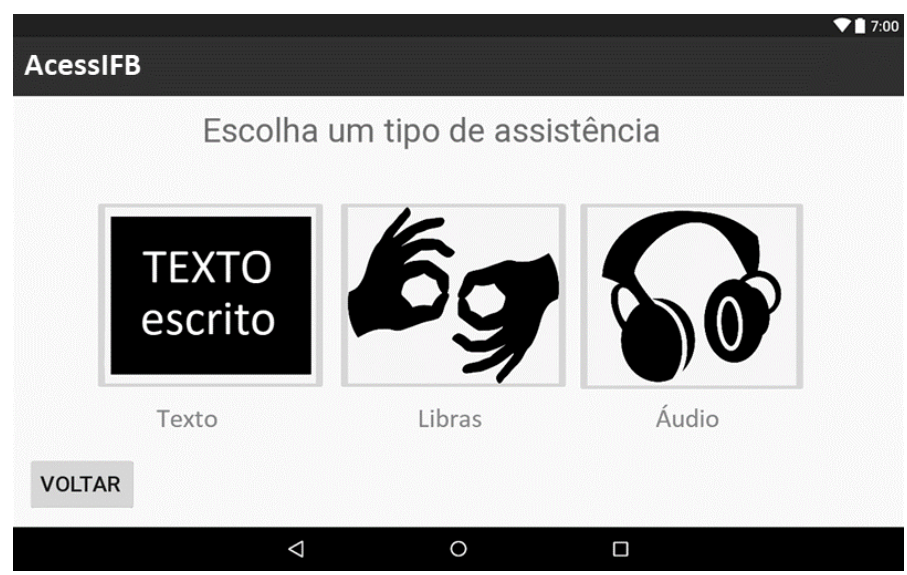

Figura 7. Pantalla principal de la propuesta actual de interfaz

Para permitir la interacción entre los componentes y el sistema, se buscó la conexión utilizando el microcontrolador Arduino (Bridi et al., 2013; Timmis, 2011). Este es un componente capaz de administrar conexiones entre equipos, como sensores, botones y otros componentes, utilizando un software desarrollado utilizando $\mathrm{C}++$. El software hace la lectura de los componentes y permite que el microcontrolador intercambie información con la tableta y la interfaz de 
usuario siendo, por lo tanto, el responsable por la gestión de la información entre los demás componentes y la tableta.

Para que los usuarios tengan un rápido conocimiento del equipo y una mayor interacción, el tótem posee sensores de infrarrojos capaces de enviar información al software presente en Arduino; éste procesa los datos para enviarlos a la tableta. A su vez, este procesa los datos e informa al usuario en formato de audio, utilizando los altavoces y avisándole, por ejemplo, que debe acercarse para obtener información sobre la cámara.

Todas estas características permiten más autonomía a quienes buscan información diversa referente a los campi del IFB, sin necesidad de un intérprete de libras ni de otra persona, como la recepcionista. Esto se logra, primero, por la identificación del usuario y continúa cuando el usuario se acerca y puede seleccionar, a través de los botones, el tipo de ayuda (visual, audio o símbolos), o también utilizando un comando de voz cuando sea solicitado por el tótem.

Es importante resaltar que, para proseguir con el desarrollo de la interfaz, el proyecto prevé la participación de un equipo multidisciplinario, compuesto por estudiantes de cursos del IFB volcados al área de programación y desarrollo de sistemas; traductor e intérprete de libras y docentes del mismo lenguaje, que componen el grupo de investigación institucional, para contribuir con la elaboración de los vídeos en libras; revisor de lengua portuguesa para apoyar el texto escrito de las correcciones; equipo de asistencia al alumno y al bibliotecario, para contribuir con la información pertinente a las respectivas áreas; equipo pedagógico y coordinaciones de curso para auxiliar en la divulgación de ofertas, vacantes y cronograma de selección de los campi.

\section{Resultados}

La aplicación se desarrolló utilizando el entorno de desarrollo gratuito Android Studio versión 2.3.3. Para crear la presentación de la aplicación durante el evento mencionado se utilizó la tableta de la coordinadora del proyecto. Ante la imposibilidad de adquirir un soporte físico específico (tótem), se construyó una base de apoyo con la ayuda de la impresora 3D del Campus Taguatinga.

Aunque estaba previsto el uso de sensores para detectar la presencia de personas a medida que llegaban cerca del equipo, solo fue desarrollada parte de esta funcionalidad y el software escrito fue cargado en la placa de desarrollo Arduino. No fue implementada la utilización de botones con escritura táctil, para que personas con discapacidad visual y usuarias del Braille pudieran hacer uso de los mismos; así como tampoco el uso del auricular para que el visitante pudiera acceder a la información que el dispositivo posee. De este modo, el uso de los sensores, botones, auriculares y el Arduino fueron destinados a una próxima versión del dispositivo, ya que es necesario un mayor tiempo para desarrollo y pruebas con las funcionalidades que cada uno de estos ítems proporcionará al prototipo.

Para validar la interfaz desarrollada, fueron recolectadas impresiones de las personas que visitaron el stand de FABIN durante el Conecta 2017 (Conectaif, 2017; TV IFB, 2017), considerando la opinión de estos participantes como usuario final. El registro de las sugerencias fue realizado por medio de un cuestionario impreso, completado después de cada experiencia de navegación del usuario. Entre aquellos que visitaron el stand, registramos la presencia de profesores y estudiantes sordos, intérpretes de libras, además del público en general que visitó el evento, cuyas percepciones ayudaron a definir mejoras, desde el tamaño de la fuente, el contraste de la pantalla,

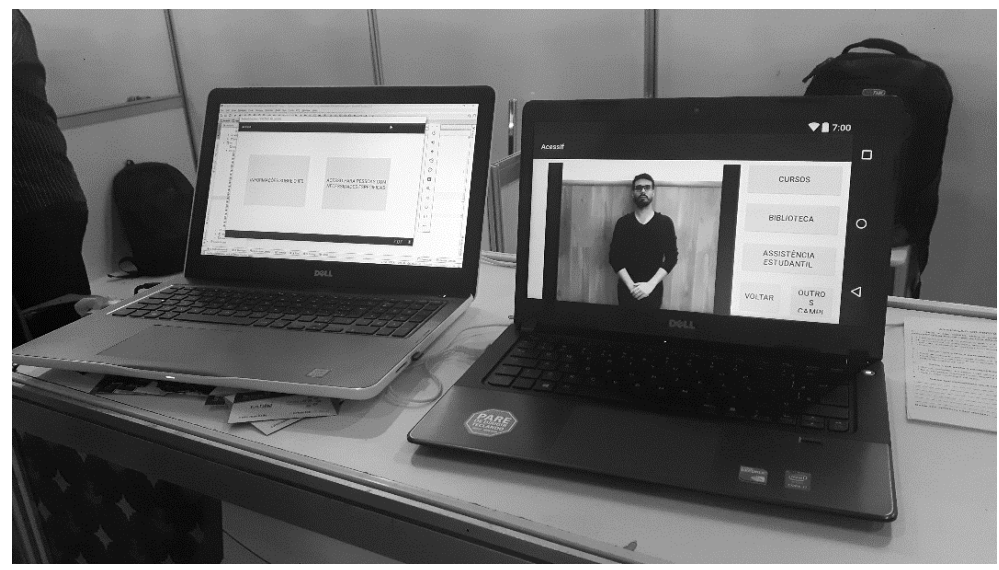

Figura 8. Pantalla principal de la primera propuesta de interfaz 
hasta la sugerencia para cambiar algunas señales utilizadas en el vídeo en libras atendiendo a diferencias locales y regionales. La Figura 8 muestra el prototipo que fue presentado en el stand durante el evento mencionado.

Los resultados muestran que, aunque el desarrollo de la interfaz no ha sido finalizado del modo previsto, la continuidad del proyecto y la comprensión del público identificado como potencial usuario puede resultar en la concreción de una herramienta muy útil como propuesta de interacción para el suministro de información de forma accesible.

\section{Agradecimientos}

A la Pro-rectoría de Investigación e Innovación del Instituto Federal de Brasilia por la financiación por medio de la Llamada Pública para la Fábrica de Ideas Innovadoras, lo que hizo posible la elaboración de la versión de este prototipo y su presentación durante el ConectaIF 2017.

\section{Referencias}

Bridi, E., Gianesini, B. M., Bianchi, E. C., Vilerá, K. V., Dias, R. R., Capriata, V. y Martins, W. K. A. G. (2013). Oficina de Arduíno como ferramenta interdisciplinar no curso de engenharia elétrica da UFMT: A experiência do PET-Elétrica. En XLI Congresso Brasileiro de Educação em Engenharia (COBENGE 2013).

CONECTAIF (2017). Encontro de Educação Profissional, Cientifica e Tecnológica. Disponible en https://conectaif.ifb.edu.br/ attachments/article/308/ConectaIF_2017.pdf

Instituto Federal de Brasília (2017a). IFB em números. Accedido 28 de noviembre de 2017, disponible en http://ifbemnumeros.ifb.edu.br Instituto Federal de Brasília (2017b). Site Institucional. Accedido 28 de noviembre de 2017, disponible en http://www.ifb.edu.br/

Instituto Nacional de Educação de Surdos - INES (2017). Fórum Bilíngue Libras-Português. Accedido 28 de noviembre de 2017, disponible en http:/www.ines.gov.br/noticias/239-forum-bilingue-do-ines-discute-surdez-educacao-e-linguabrasileira-de-sinais

Ministério do Planejamento Orçamento, Secretaria de Logística, \& Tecnologia da Informação (2014). eMAG - Modelo de Acessibilidade em Governo Eletrônico. Disponible en https://www.governodigital.gov.br/documentos-e-arquivos/ eMAGv31.pdf

Presidência da República (2015). Lei No 13.146 de 6 de Julho de 2015. Accedida 28 de noviembre de 2017, disponible en http:// www.planalto.gov.br/ccivil_03/_ato2015-2018/2015/lei/113146.htm

Ramalho, F., Hamad, H. y Guimarães, Í. J. B. (2016). Comportamento informacional dos discentes deficientes visuais da Universidade Federal da Paraíba - UFPB. Informação \& Informação, 21(1), 230-256. https://doi.org/10.5433/1981$8920.2016 \mathrm{v} 21 \mathrm{n} 1 \mathrm{p} 230$

Timmis, H. (2011). Practical Arduino Engineering. Berkeley, CA: Apress. https://doi.org/10.1007/978-1-4302-3886-7

TV IFB. (2017). Fabin - AcessIFB. Disponible en https://www.youtube.com/watch?v=Qxml-vSRlLk\&index=20\&list=PL_ oCE450MW3QM55YSNx57CiK4cAA5oBDv

\section{Sobre los autores}

\section{Sylvana Karla da Silva de Lemos Santos.}

Es ingeniera electrónica y tecnóloga en Análisis de sistemas, maestría en Ingeniería mecánica y estudiante de doctorado en Ciencia de la información. Es docente de Informática en el Instituto Federal de Brasilia, Campus Brasília, Brasil.

\section{Joaquim Alves Costa Júnior.}

Es ingeniero de Control y automatización y estudiante graduado de Ciencia de la computación en el Instituto Federal de Brasilia, Campus Taguatinga, Brasil.

\section{Jaspion Leone Rocha.}

Es graduado en Letras libras, especialista en Educación especial. Traductor e intérprete de Lengua de señales brasileña en el Instituto Federal de Brasilia, Rectoría, Brasil.

\section{Danilo Freire Holanda de Paiva.}

Es estudiante graduado de Ciencia de la computación en el Instituto Federal de Brasilia, Campus Taguatinga, Brasil. 